Original paper

\title{
A new model to predict response to direct-acting antiviral therapy in decompensated cirrhotics due to hepatitis $C$ virus
}

\author{
Prasanta Debnath', Sanjay Chandnani', Pravin Rathi', Sujit Nair', Parmeshwar Junare', Suhas Udgirkarr', Anupam Singh², \\ Qais Contractor ${ }^{1}$
}

${ }^{1}$ TNMC \& BYL Nair Charitable Hospital, Mumbai, India

2Santosh Medical College, Ghaziabad, India

\begin{abstract}
Aim of the study: Decompensated hepatitis C virus (HCV) cirrhosis is a difficult to treat cohort, and there is no gold standard predictor of response to direct-acting antiviral (DAA) therapy. We conducted this study to look for factors responsible for improvement in post-therapy status, i.e. attainment of Child-Turcotte-Pugh (CTP) class A from $B$ or $C$, and devise a new model to predict post-therapy response.

Material and methods: Prospective analysis of data from decompensated HCV cirrhotics was done and association of each parameter with patient outcomes at 36 weeks after treatment was assessed.

Results: 34 patients (54.8\%) attained CTP class A after treatment. Factors that were independently associated with disease outcome included albumin (odds ratio $[O R]=4.84,95 \%$ confidence interval $[\mathrm{Cl}]$ : 1.43-20.15, $p=0.018$ ), alanine transaminase (ALT) (OR $=1.02,95 \% \mathrm{Cl}: 1-1.04, p=0.049)$, bilirubin (OR $=0.41,95 \% \mathrm{Cl}$ : $0.2-0.75, p=0.007$ ) and estimated glomerular filtration rate (eGFR) (OR $=1.03,95 \% \mathrm{Cl}: 1.0-1.06, p=0.045)$. On multivariate analysis, bilirubin was significantly associated with treatment outcome $(O \mathrm{R}=0.28,95 \% \mathrm{Cl}$ : $0.1-0.64, p=0.006$ ). A composite model was devised using demographic, biochemical, and clinical features, which has sensitivity, specificity, positive predictive value, negative predictive value and accuracy of $67.86 \%$, $79.41 \%, 73.08 \%, 75 \%$, and $73.63 \%$ respectively in predicting response to therapy. Only $7.6 \%$ of patients with a Model for End-Stage Liver Disease (MELD) score $>15$ and none of the patients with CTP class C met the primary end-point of our study.

Conclusions: $55 \%$ of our cohort met the primary end-point at 36 weeks. Patients with CTP class C and a MELD score $>15$ should be referred for liver transplantation followed by DAA therapy. Our model was good at predicting improvement in post-therapy liver function.
\end{abstract}

Key words: hepatitis C virus, direct-acting antivirals, decompensated cirrhosis, BE3A score.

Address for correspondence:

Dr. Prasanta Debnath, TNMC \& BYL Nair Charitable Hospital, Mumbai, India, e-mail: prasantad89@gmail.com

\section{Introduction}

Chronic hepatitis C virus (HCV) infection progresses slowly and leads to cirrhosis in 20-30 years. After the development of cirrhosis, the risk of progression of liver damage to hepatic decompensation is approximately 3-6\%/year [1]. Patients with decompensated cirrhosis, Child-Turcotte-Pugh (CTP) class B or C, have a high risk of developing portal hypertension-related complications such as ascites, jaundice, variceal bleeding, or hepatic encephalopathy in due course of time. However, oral direct-acting antivirals (DAAs) have markedly revolutionized the management of patients with chronic HCV infection. In patients with compensated cirrhosis, sustained virological response (SVR) has been more than $90 \%$ and around $80 \%$ in cas- 
es of decompensated cirrhosis [2-5]. Successful HCV treatment has been shown to lower the risk of hepatic failure, hepatocellular carcinoma (HCC), liver-related deaths, and all-cause mortality. Approximately one-quarter of patients with compensated cirrhosis no longer have liver stiffness in the cirrhotic range within 1 year of SVR [6]. However, in cases of decompensated cirrhosis, it is presently not clear whether viral clearance results in a lasting improvement of signs of liver decompensation. Although the definitive treatment for patients with decompensated cirrhosis is liver transplantation (LT), recent studies have shown meaningful biochemical and clinical improvement in patients with HCV-related decompensated cirrhosis on DAA therapy. Patients listed for LT due to decompensated cirrhosis had improvement in their liver function to an extent that some of them were inactivated from the waiting list and eventually delisted $[7,8]$.

In the past, it has been observed that when LT candidates with decompensated cirrhosis due to hepatitis $B$ virus (HBV) related liver disease were treated with nucleo(s/t)ide analogues (NUC), almost one third of these patients were eventually delisted while on NUC therapy and their clinical improvement could be maintained for up to 5 years [9]. Patients with untreated hepatitis $\mathrm{C}$ or failed treatment who underwent LT can have a significantly accelerated disease course compared to a non-LT setting. Between 10\% and 30\% developed cirrhosis within 5 years from LT and $40 \%$ presented signs of liver decompensation within 1 year from the diagnosis of recurrent cirrhosis [10-12]. Due to the effectiveness of DAA therapy the prevalence of HCV infection is expected to decline but hepatocellular carcinoma and liver-related deaths are expected to continue rising worldwide until 2030 [13].

Few studies have looked for factors that are associated with response to DAA therapy in patients with HCV-related liver disease. Ahmed et al. found that factors responsible for poor response to DAAs were older age, cirrhosis, especially CTP class B, and low platelet count found in an Egyptian study using sofosbuvir and daclatasvir for genotype 4 [14]. In another study reduced response rates occurred more frequently in treatment-experienced patients, those with advanced cirrhosis, HCV genotypes 3 or la infections, elevated serum HCV-RNA, poor drug adherence or premature drug discontinuation [15]. Recently a new score (BE3A) has been devised using the sum of five factors, namely body mass index (BMI) $<25 \mathrm{~kg} / \mathrm{m}^{2}$, absence of encephalopathy, absence of ascites, alanine transaminase (ALT) $>60 \mathrm{IU} / \mathrm{l}$, and albumin $>3.5 \mathrm{~g} / \mathrm{dl}$, which were identified to be associated with clinical recovery from decompensated cirrhosis after DAA treatment [16]. Patients with a BE3A score of 4 have a $75 \%$ chance of recovering to CTP class A after DAA treatment.

There is scanty literature regarding predictive factors of response in cases of decompensated HCV-related cirrhosis. Hence, in this study, we looked for factors which are responsible for the response to DAA therapy and achieving a clinically meaningful treatment benefit which was defined as reduction to CTP class A (i.e. down-staging from CTP class B to A or C to A) sustained until the end of follow-up. We also devised a new model and compared it with the validated BE3A score.

\section{Material and methods}

\section{Patients}

Patients treated from January 2016 to August 2018 were prospectively included. Written consent was taken from all patients. The study was approved by the Institute's Ethics Committee. All patients who were age 18 years or older and having chronic HCV infection of genotype 1-6 and decompensated cirrhosis (CTP B or $\mathrm{C}$ ) were included. Abdominal ultrasonography and biochemical tests including liver function tests and serum $\alpha$-fetoprotein (AFP) were done in all patients. A triple-phase computed tomography (CT) scan of the abdomen was considered for patients with raised serum AFP. Patients diagnosed to have HCC were excluded from the study. Patients having co-infection with human immunodeficiency virus (HIV), hepatitis $B$ virus (HBV) and previous exposure to DAAs were also excluded. Moreover, patients with any of the following baseline laboratory parameters were also excluded: platelets $<30,000 / \mathrm{mm}^{3}$, ALT, aspartate aminotransferase (AST), or alkaline phosphatase (ALP) more than 10 times the upper limit of normal or estimated glomerular filtration rate (eGFR) $<30 \mathrm{ml} / \mathrm{min} / 1.73 \mathrm{~m}^{2}$.

We excluded 13 out of 75 patients with decompensated cirrhosis because they had: HBV co-infection (4 patients), HCC (5 patients), previous exposure to DAA (2 patients) and death unrelated to cirrhosis (2 patients). The remaining 62 patients were included in our study. Written informed consent was taken from each patient.

\section{Clinical, anthropometric and laboratory evaluation}

Independent variables based on clinical relevance to cirrhosis and liver decompensation were noted, which included weight, height, BMI, complete blood 
count (CBC), liver function test (LFT) including total bilirubin, ALT, AST, albumin, international normalized ratio (INR), serum creatinine and eGFR. Quantitative HCV-RNA using the TaqMan Real-Time PCR technique and HCV genotype were analyzed for each patient. Clinical variables such as presence of ascites, encephalopathy and any evidence of gastrointestinal bleeding were also noted. All variables were categorized to facilitate clinical interpretation. Binary variables were adopted for presence or absence of ascites and encephalopathy. Conventional cut-off points for the Indian population were used for BMI. Body weight was adjusted based on the severity of ascites and the presence of pedal swelling before measuring BMI. A percentage of body weight was subtracted from total body weight based on the severity of ascites (mild $5 \%$, moderate $10 \%$, severe $15 \%$ ), with an additional $5 \%$ subtracted if bilateral pedal edema was present.

The BE3A score was calculated by the sum of its 5 components; each had 1 point when the criterion was met.

The Model for End-Stage Liver Disease (MELD) score and CTP score were calculated for each patient using appropriate variables.

DAA therapy was considered for each patient. Sofosbuvir $400 \mathrm{mg}$ along with daclatasvir $60 \mathrm{mg}$ or velpatasvir $100 \mathrm{mg}$ with or without ribavirin was considered in patients with HCV genotype 2 or 3 infection, whereas sofosbuvir $400 \mathrm{mg}$ along with ledipasvir $90 \mathrm{mg}$ with or without ribavirin was considered for $\mathrm{HCV}$ infection with genotype 1,4,5 or 6 infection [17]. Daclatasvir was used initially until July 2017 in the study period when velpatasvir was not available in India. Ribavirin was used according to body weight $(1000 \mathrm{mg} /$ day if weight $<75 \mathrm{~kg}, 1200 \mathrm{mg} /$ day if weight $>75 \mathrm{~kg}$ ). Ribavirin was started at a low dose for each patient $(600 \mathrm{mg})$ and gradually increased at a dose of $200 \mathrm{mg} /$ week to the target dose if tolerated based on hemoglobin values. In the course of treatment, if hemoglobin dropped below $10 \mathrm{~g} / \mathrm{dl}$, the ribavirin dose was reduced by $200 \mathrm{mg}$ and was stopped in patients whose hemoglobin dropped below $8.5 \mathrm{~g} / \mathrm{dl}$. Ribavirin was not used in those whose baseline hemoglobin was $<10 \mathrm{~g} / \mathrm{dl}$ [18].

Treatment was continued for 12 weeks in those in whom ribavirin was used and for 24 weeks for the rest of the patients.

Routine investigations including CBC, LFT, creatinine and INR along with clinical assessment for the presence of ascites and encephalopathy were done at each patient's follow-up. Every patient was followed up after two weeks of starting therapy and then every 4 weeks. Patients in the ribavirin group were assessed with $\mathrm{CBC}$ weekly for 4 weeks until the target dose was achieved, then they were monitored every two weeks to look for hematological side-effects.
Quantitative HCV-RNA was analyzed at 4 weeks to look for a rapid virologic response (RVR) then at end of treatment, and finally at 12 weeks after treatment to look for SVR. CTP and MELD scores were calculated at every follow-up visit.

All included patients were followed up for 36 weeks after treatment. Any decompensating events while on treatment or in the post-treatment period were recorded for each patient.

\section{Statistical analysis}

Descriptive and inferential statistical analysis was carried out in the present study. Results on continuous measurements are presented as mean $\pm \mathrm{SD}$ (min-max) and results on categorical measurements are presented as numbers (\%). Student's $t$-test (two-tailed, independent) was used to determine the significance of study parameters on a continuous scale between two groups (Intergroup analysis) on metric parameters. Leven's test for homogeneity of variance was performed to assess the homogeneity of variance.

Chi-square/Fisher's exact test was used to find the significance of study parameters on a categorical scale between two or more groups, the non-parametric setting for qualitative data analysis. Fisher's exact test was used for a small sample size.

Univariate analysis was performed to estimate odds ratio (OR) for age, sex, components of CTP score, albumin, ALT, eGFR, as well as its components. Multivariate analysis was also done for the same variables to estimate $\mathrm{OR}$. The results were used to generate a model for predicting response to DAA therapy in decompensated HCV-related cirrhosis. The newly devised model was then compared with the validated BE3A score to evaluate its performance in a real-life scenario.

Statistical software: SPSS 22.0, and R environment ver.3.2.2 were used for the analysis of data and Microsoft Word and Excel were used to generate graphs, tables, etc.

\section{Results}

\section{Demographic profile}

Sixty-two patients with decompensated cirrhosis due to HCV were included. The treatment options available, along with the side-effect profile of existing drugs, were explained to all patients.

\section{Patient characteristics}

All were treatment-naïve cases of decompensated cirrhosis due to HCV infection. The median age 
Table 1. Demographic and baseline characteristics of patients

\begin{tabular}{|c|c|}
\hline Variables & Values \\
\hline Age, median (range) & $52(42-80)$ \\
\hline Male, $n(\%)$ & $36(58.1 \%)$ \\
\hline \multicolumn{2}{|l|}{$\operatorname{BMl}\left(\mathrm{kg} / \mathrm{m}^{2}\right), n(\%)$} \\
\hline$<23.5$ & $46(74.2 \%)$ \\
\hline$>23.5$ & $16(25.8 \%)$ \\
\hline HCV RNA (IU/ml), median (range) & $\begin{array}{c}2,53,000 \\
(2,300-3,38,60,000)\end{array}$ \\
\hline \multicolumn{2}{|l|}{ Genotype, $n(\%)$} \\
\hline 1 & $11(17.7 \%)$ \\
\hline 2 & 0 \\
\hline 3 & $50(80.7 \%)$ \\
\hline 4 & $1(1.6 \%)$ \\
\hline 5 & 0 \\
\hline 6 & 0 \\
\hline MELD, median (range) & $12.11 \pm 3.91$ \\
\hline \multicolumn{2}{|l|}{ MELD classes, $n(\%)$} \\
\hline$<10$ & $21(33.9 \%)$ \\
\hline $10-14$ & $28(45.1 \%)$ \\
\hline$\geqslant 15$ & $13(21 \%)$ \\
\hline Child-Pugh Score, mean ( $\pm S D$ ) & $8.11 \pm 1.05$ \\
\hline \multicolumn{2}{|l|}{ CTP classes, $n(\%)$} \\
\hline A & 0 \\
\hline B & $55(88.7 \%)$ \\
\hline C & $7(11.3 \%)$ \\
\hline \multicolumn{2}{|l|}{ Ascites, $n(\%)$} \\
\hline None & 0 \\
\hline Mild-moderate & $49(79 \%)$ \\
\hline Severe & $13(21 \%)$ \\
\hline \multicolumn{2}{|l|}{ Encephalopathy, $n$ (\%) } \\
\hline None & $59(95.2 \%)$ \\
\hline West Haven grade 1, 2 & $3(4.8 \%)$ \\
\hline West Haven grade 3,4 & 0 \\
\hline \multicolumn{2}{|l|}{ DAA treatment, $n(\%)$} \\
\hline $\mathrm{SOF}+\mathrm{DAC} \pm \mathrm{RIB}$ & 27 (43.5\%) \\
\hline $\mathrm{SOF}+\mathrm{LED} \pm \mathrm{RIB}$ & $11(17.8 \%)$ \\
\hline$S O F+V E L \pm R I B$ & $24(38.7 \%)$ \\
\hline \multicolumn{2}{|l|}{ BE3A score } \\
\hline 1 & $12(19.4 \%)$ \\
\hline 2 & $32(51.6 \%)$ \\
\hline 3 & $16(25.8 \%)$ \\
\hline 4 & $2(3.2 \%)$ \\
\hline 5 & 0 \\
\hline
\end{tabular}

MELD - Model for End-stage Liver Disease, DAA - direct-acting antivirals, SOF- sofosbuvir, RIB - ribavirin, LED - ledipasvir, DAC - daclatasvir, VEL - velpatasvir of our study population was 52 years (42-80), with males constituting 36 (58.1\%) of all. Out of 62 patients, 55 (88.7\%) patients belonged to CTP class B and the remaining $7(11.3 \%)$ had CTP class C. Genotype 3 was the most common genotype causing $\mathrm{HCV}$ infection in our study population, with 50 cases $(80.7 \%)$, followed by genotype 1 in 11 (17.7\%) and genotype 4 in $1(1.6 \%)$. The median HCV-RNA count in our patient population was $2,53,000(2,300-3,38,60,000) \mathrm{IU} / \mathrm{ml}$.

Demographic and baseline characteristics are shown in Table 1.

\section{Virological outcomes}

Virological clearance, defined as HCV-RNA < lower limit of quantification with a detection threshold of $15 \mathrm{IU}$, was observed in $62(100 \%)$ patients after 4 weeks of therapy $(\mathrm{RVR}=100 \%)$ and in $60(96.7 \%)$ patients after 12 weeks of treatment. Two patients failed to clear HCV RNA after 12 weeks of treatment. Both of them were on sofosbuvir and velpatasvir and treatment was continued for 24 weeks. Ribavirin could not be used in either because of anemia.

The number of patients receiving sofosbuvir combination therapy with daclatasvir, ledipasvir or velpatasvir was 27 (43.5\%), 11 (17.8\%) and 24 (38.7\%) respectively. Ribavirin was used in $24(38.7 \%)$ patients with no contraindication to it along with sofosbuvir combination therapy.

The total number of responders, i.e. attainment of CTP class A state, was 34 (54.8\%) and 28 (45.2\%) were non-responders.

The total number of responders was higher in patients with a higher BE3A score (Table 1). The number of responders was 3 (25\%), 14 (43.75\%), 15 (93.75\%) and $2(100 \%)$ with a BE3A score of $1,2,3$ and 4 respectively (Fig. 1).

\section{Impact of DAA on biochemical parameters}

The pre-treatment and post-treatment values of various biochemical parameters are tabulated in Table 2 .

\section{Clinical profile of patients}

\section{Clinical outcome}

Death: Two patients (3.2\%) died during the study period. One death was recorded in the 2 nd week of treatment in a patient who had advanced decompensated liver disease. The other one was non-compliant to therapy with decompensation and acute kidney injury and died during the $4^{\text {th }}$ week of therapy. 


\section{Transplant}

None of the patients in our study group underwent liver transplantation.

\section{Decompensating events}

Out of 60 patients who attained SVR 12, 36 (58\%) patients did not have any decompensating events in the follow-up period, whereas 24 (38.7\%) patients were noted to have presented with one or more decompensating events in the follow-up period.

Two patients who failed to achieve SVR12 had one or more decompensating events in the follow-up period.

\section{Impact of DAA on clinical parameters}

Before DAA therapy mild to moderate ascites was seen in $49(79 \%)$ patients and severe ascites in $13(31 \%)$ patients. After treatment 45 (72.6\%) patients had complete resolution of ascites, $16(25.8 \%)$ patients had persistent mild to moderate ascites and one (1.6\%) patient had severe ascites.

In our study population, 3 (4.8\%) patients had a history of encephalopathy (West Haven grade 1 and 2) before treatment. No patients had a history of West Haven grade 3 or 4 encephalopathy. After treatment $1(1.6 \%)$ had a history of encephalopathy for which hospitalization was required.

\section{Impact on CTP score}

In the pre-treatment period, the numbers of patients in CTP class B and C were 55 (88.7\%) and $7(11.3 \%)$ respectively. After DAA therapy there was a marked improvement in CTP score. The number of patients in CTP class A, B, and C were found to be $34(54.8 \%), 25(40.3 \%)$ and $3(4.8 \%)$ respectively (Fig. 2 ).

\section{Impact on MELD score}

The number of patients with a MELD score $<10$, $10-15$ and $>15$ in the pre-treatment period were $21(33.9 \%), 28(45.1 \%)$ and $13(21 \%)$ respectively. In the post-treatment period, the number of patients with a MELD score < 10 increased to $23(37.1 \%)$, and that in $10-15$ and $>15$ groups was $26(48.4 \%)$ and $9(14.5 \%)$ respectively (Fig. 3).

Delta MELD was calculated using the difference in MELD values at baseline and post-treatment period. The mean value of delta MELD in treatment responders, i.e. CTP class A patients, and non-responders was $1.35 \pm 1.81$ and $0.46 \pm 2.87$ respectively and was not

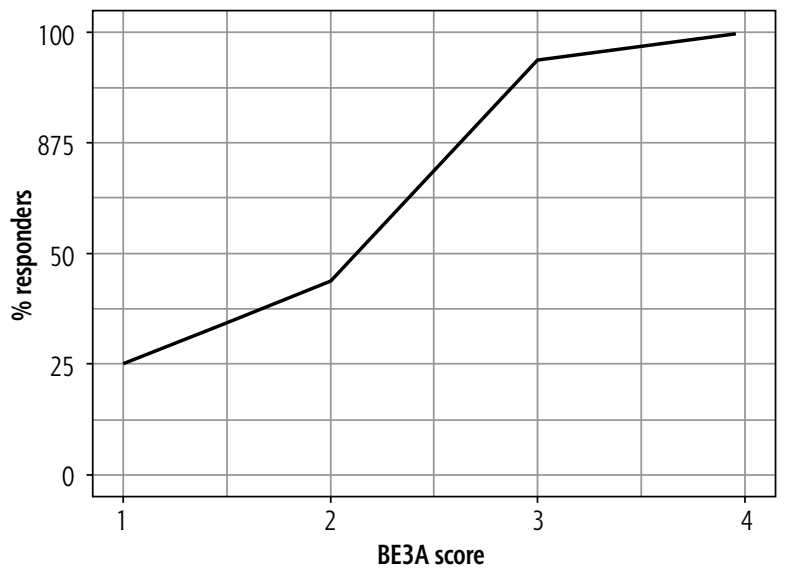

Fig. 1. Line graph showing the relationship of $B E 3 A$ score to response, i.e. attainment of CTP A state, in post-treatment and follow-up period

Table 2. Biochemical parameters in pre-treatment and post-treatment period

\begin{tabular}{lcc}
\hline Parameter & $\begin{array}{c}\text { Pre-treatment value } \\
(\text { mean } \pm \text { SD) }\end{array}$ & $\begin{array}{c}\text { Post-treatment value } \\
\text { (mean } \pm \text { SD) }\end{array}$ \\
\hline Hemoglobin $(\mathrm{g} / \mathrm{dl})$ & $9.51 \pm 1.85$ & $11.54 \pm 1.20$ \\
\hline Platelet count $\left(10^{6} / \mathrm{ml}\right)$ & $107.77 \pm 37.02$ & $126 \pm 34.18$ \\
\hline TLC $\left(10^{3} / \mathrm{ml}\right)$ & $6,250 \pm 1603.23$ & $8,500 \pm 2478.68$ \\
\hline Bilirubin $(\mathrm{mg} / \mathrm{dl})$ & $1.7 \pm 0.94$ & $1.69 \pm 0.94$ \\
\hline AST $(\mathrm{U} / \mathrm{l})$ & $65.27 \pm 35.04$ & $46.78 \pm 28.58$ \\
\hline ALT $(\mathrm{U} / \mathrm{l})$ & $53.53 \pm 32.97$ & $42.52 \pm 30.71$ \\
\hline ALP $(\mathrm{IU} / \mathrm{l})$ & $133 \pm 35.6$ & $142 \pm 32.9$ \\
\hline Total protein $(\mathrm{g} / \mathrm{l})$ & $6.23 \pm 0.59$ & $6.48 \pm 0.74$ \\
\hline Albumin $(\mathrm{g} / \mathrm{l})$ & $2.86 \pm 0.46$ & $3.15 \pm 0.39$ \\
\hline INR & $1.28 \pm 0.29$ & $1.26 \pm 0.23$ \\
\hline Creatinine $(\mathrm{mg} / \mathrm{dl})$ & $1.12 \pm 0.41$ & $0.92 \pm 0.29$ \\
\hline
\end{tabular}

statistically significantly different $(p=0.214)$. Similarly, delta albumin was calculated from the difference in serum albumin values in the pre- and post-treatment periods. Mean value in responders and non-responders was $0.34 \pm 0.39 \mathrm{~g} / \mathrm{dl}$ and $0.23 \pm 0.43 \mathrm{~g} / \mathrm{dl}$ respectively $(p=0.147)$.

On univariate analysis, serum bilirubin $(\mathrm{OR}=0.41$, 95\% CI: 0.20-0.75), albumin ( $\mathrm{OR}=4.84$, 95\% CI: 1.43 20.15) and eGFR (OR $=1.03,95 \% \mathrm{CI}: 1.0-1.06)$ and on multivariate analysis only serum bilirubin $(\mathrm{OR}=$ 0.28, 95\% CI: 0.1-0.64) were found to be predictors of attainment of CTP class A after DAA therapy in decompensated HCV cirrhosis patients (Table 3).

\section{Deriving our new model}

A new model was devised with the parameters age, gender, platelet, serum bilirubin, ALT, albumin, INR, 


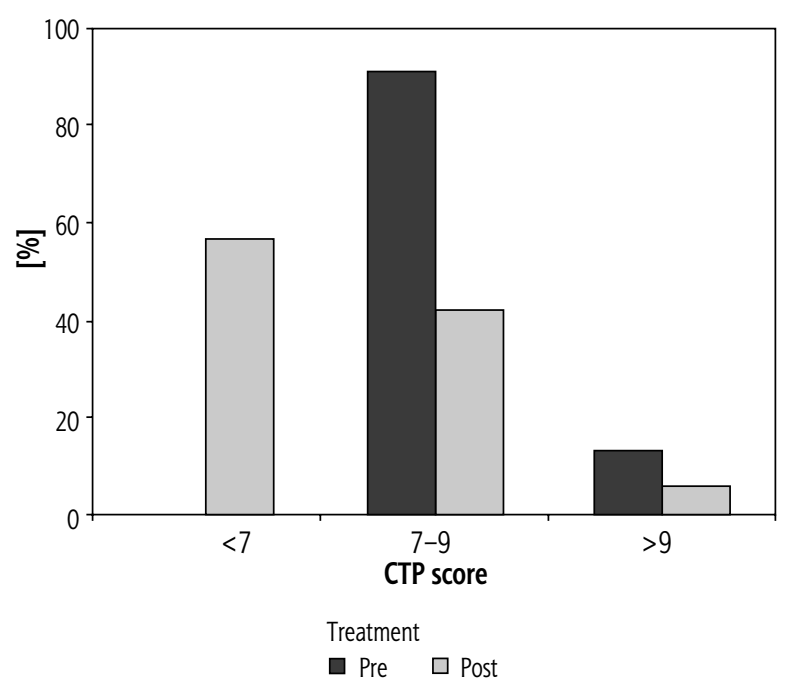

Fig. 2. Bar diagram showing patients with CTP class A (score < 7), B (score 7-9) and $C$ (score $>9$ ) in pre- and post-treatment period

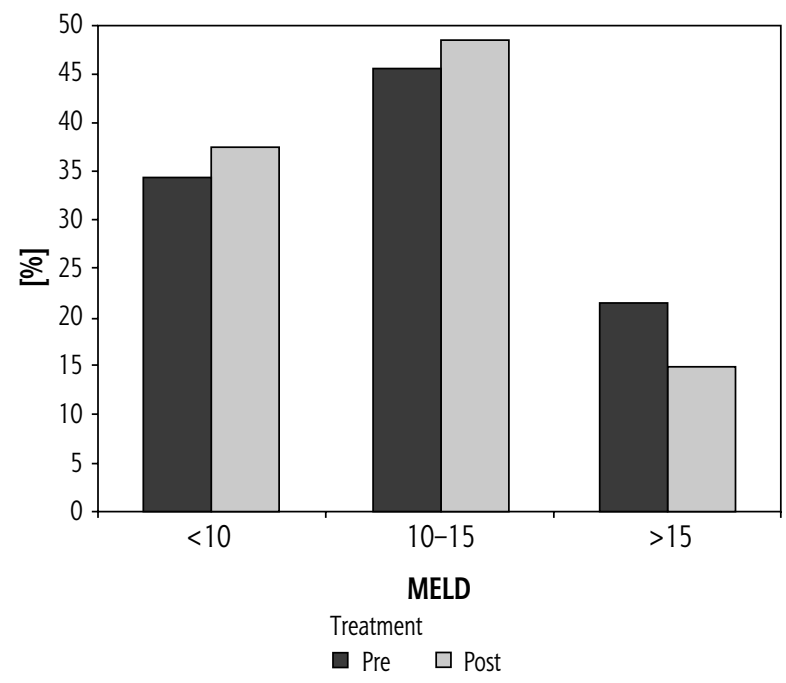

Fig. 3. Bar diagram showing patients with baseline MELD score $<10,10-15$ and $>15$ in pre- and post-treatment period

eGFR and clinical variables - the presence of ascites and encephalopathy - for predicting response to DAA therapy and attainment of CTP class A after treatment.

We fitted a logistic model (estimated using ML) to predict responders with age, gender, serum bilirubin, albumin, INR, ALT, eGFR and presence of ascites and encephalopathy. Standardized parameters were obtained by fitting the model on a standardized version of the dataset. Effect sizes were labeled following Chen's (2010) recommendations.

The probability was calculated from the odds ratio using the following equation:

Probability $=$ Odds $/ 1+$ Odds,

where the odds for achieving CTP A status was expressed as:
Table 3. Summary table of logistic regression of responder against predictors

\begin{tabular}{|c|c|c|c|c|c|c|}
\hline \multirow[t]{2}{*}{ Variables } & \multicolumn{3}{|c|}{ Univariate model } & \multicolumn{3}{|c|}{ Multivariate model } \\
\hline & OR & $95 \% \mathrm{Cl}$ & $p$-value & OR & $95 \% \mathrm{Cl}$ & $p$-value \\
\hline Age (years) & 0.97 & $0.92-1.01$ & 0.19 & 0.98 & $0.91-1.05$ & 0.647 \\
\hline \multicolumn{7}{|l|}{$<65$} \\
\hline \multicolumn{7}{|l|}{$>65$} \\
\hline Sex & 0.97 & $0.92-1.01$ & 0.190 & 0.98 & $0.91-1.05$ & 0.647 \\
\hline \multicolumn{7}{|l|}{ Female } \\
\hline \multicolumn{7}{|l|}{ Male } \\
\hline ALT & 1.02 & $1.00-1.04$ & 0.049 & 1.04 & $1.00-1.08$ & 0.074 \\
\hline \multicolumn{7}{|c|}{$<1.5 \times$ ULN } \\
\hline \multicolumn{7}{|c|}{$>1.5 \times$ ULN } \\
\hline $\begin{array}{l}\text { Bilirubin } \\
\text { (mg/dl) }\end{array}$ & 0.41 & $0.20-0.75$ & 0.007 & 0.28 & $0.10-0.64$ & 0.006 \\
\hline \multicolumn{7}{|l|}{$<2$} \\
\hline \multicolumn{7}{|l|}{$2-3$} \\
\hline \multicolumn{7}{|l|}{$>3$} \\
\hline INR & 0.35 & $0.05-1.96$ & 0.244 & 0.41 & $0.03-4.99$ & 0.494 \\
\hline \multicolumn{7}{|l|}{$<1.7$} \\
\hline \multicolumn{7}{|l|}{$1.7-2.3$} \\
\hline \multicolumn{7}{|l|}{$>2.3$} \\
\hline $\begin{array}{l}\text { Albumin } \\
(\mathrm{g} / \mathrm{dl})\end{array}$ & 4.84 & $\begin{array}{l}1.43- \\
20.15\end{array}$ & 0.018 & 7.02 & $\begin{array}{l}1.01- \\
82.21\end{array}$ & 0.076 \\
\hline \multicolumn{7}{|l|}{$>3.5$} \\
\hline \multicolumn{7}{|l|}{$2.8-3.5$} \\
\hline \multicolumn{7}{|l|}{$<2.8$} \\
\hline Ascites & 0.64 & $0.18-2.21$ & 0.481 & 0.92 & $0.19-4.60$ & 0.914 \\
\hline \multicolumn{7}{|l|}{ No } \\
\hline \multicolumn{7}{|l|}{ Yes } \\
\hline $\begin{array}{l}\text { Encepha- } \\
\text { lopathy }\end{array}$ & 0.39 & $0.02-4.33$ & 0.457 & 1.04 & $0.00-2.97$ & 0.232 \\
\hline \multicolumn{7}{|l|}{ No } \\
\hline \multicolumn{7}{|l|}{ Yes } \\
\hline $\begin{array}{l}\text { eGFR } \\
(\mathrm{ml} / \mathrm{min} / \\
\left.1.73 \mathrm{~m}^{2}\right)\end{array}$ & 1.03 & $1.00-1.06$ & 0.045 & 1.04 & $0.99-1.09$ & 0.135 \\
\hline \multicolumn{7}{|l|}{$\geqslant 60$} \\
\hline$<60$ & & & & & & \\
\hline
\end{tabular}

Odds [Improvement $]=\mathrm{e}-0.018 \times[$ Age $]-0.6345$ $\times$ [Gender] $-1.2786 \times[$ Bilirubin] $-0.0891 \times$ [Ascites] $+1.9126 \times$ [Albumin] $-0.8392 \times[$ INR] $+0.0351 \times$ [ALT] $-1.9133 \times$ [Encephalopathy] $+0.036 \times[\mathrm{eGFR}]$ $-0.0018 \times$ [Platelet count] -2.7895 .

In which 1 was used for the presence of encephalopathy or ascites, and 0 for its absence respectively, for age $<65$ years 0 and $>65$ years 1 were used, for gen- 
der 0 and 1 were used for female and male respectively, with bilirubin in unit of $\mathrm{mg} / \mathrm{dl}$, albumin in unit of $\mathrm{g} / \mathrm{dl}$, ALT in unit of IU/l, eGFR in $\mathrm{ml} / \mathrm{min} / 1.73 \mathrm{~m}^{2}$ and platelet count in $10^{6} / \mathrm{ml}$.

Metrics of logistic regression using our model revealed an Akaike information criterion (AIC) and Bayesian information criterion (BIC) of 79.9 and 103 respectively.

Using the BE3A score as a predictor of treatment response, metrics of logistic regression revealed an AIC and BIC of 71.4 and 75.65 respectively.

\section{Confusion matrix of our model and BE3A score}

Our new model had sensitivity, specificity, positive predictive value, negative predictive value and accuracy of $67.86 \%, 79.41 \%, 73.08 \%, 75 \%$, and $73.63 \%$ respectively in predicting response to therapy. Similarly, with BE3A score sensitivity, specificity, positive predictive value (PPV), negative predictive value (NPV) and accuracy of predicting response to DAAs were $32.14 \%$, $91.18 \%, 75 \%, 62 \%, 61.66 \%$ respectively.

However, comparison of our model with BE3A score using ROC analysis revealed better results with our composite model, with an AUC value of 0.841 and 0.771 respectively, although DeLong's test for two correlated ROC curves revealed a statistically non-significant result ( $p=0.29)$ (Fig. 4).

\section{Discussion}

We have analyzed all 62 patients with advanced cirrhosis who underwent antiviral therapy with sofosbuvir-based DAA therapy at our center. Assessment of virologic efficacy and frequency of clinical events reflecting hepatic decompensation were studied and predictive parameters for patients at risk for decompensation were looked for. The overall SVR rate in our patient cohort was $96.8 \%$. Thirty-four (54.83\%) patients attained the primary end-point of our study, i.e. attainment and persistence of a compensated state in the post-treatment follow-up period of 36 weeks. The rate of hepatic decompensation defined as the onset of ascites, hepatic encephalopathy, upper gastrointestinal bleeding or new-onset jaundice in the follow-up period was $45.2 \%$.

At short-term follow-up (12 weeks), $47-68 \%$ of patients with decompensated HCV cirrhosis who achieved SVR did not require liver transplantation and had CTP score improvement. However, 11-24\% had worsening of CTP scores $[5,19,20]$.

In our study population, baseline serum bilirubin, ALT, MELD score and CTP class were significantly as-

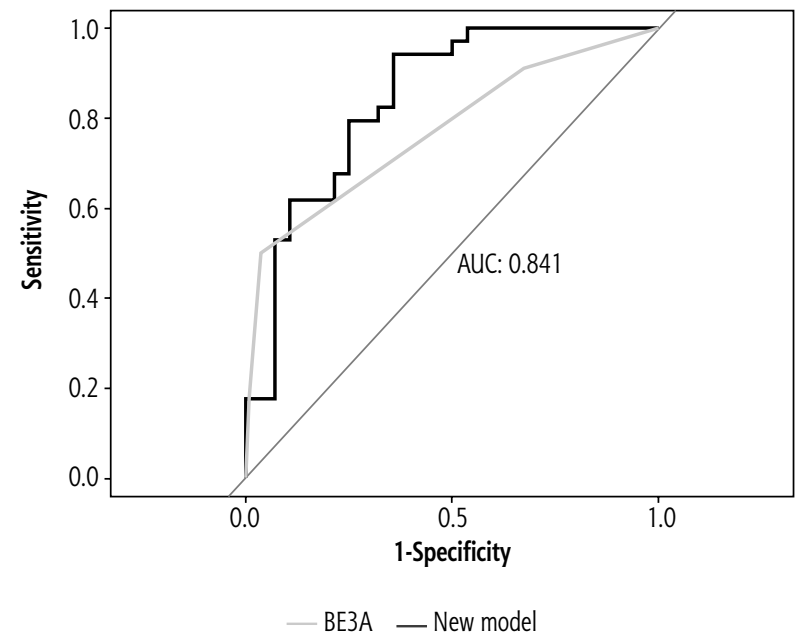

Fig. 4. Overall performance comparison of our model with BE3A score by ROC curve

sociated with response to DAA treatment. Responders, i.e. patients who had persistent CTP class A following treatment even in their follow-up period, had baseline ALT $>1.5$ times the upper limit of normal compared to non-responders. A similar finding was reported by El-Sherif et al. [16].

In patients with a MELD score of $<15,33$ out of $49(67.3 \%)$ successfully met the primary end-point of our study, whereas $16(32.7 \%)$ patients failed to do so. However, only 1 in 13 (7.6\%) patients with a MELD score $>15$ attained a state of compensated liver disease after DAA therapy. In CTP class B, 34/55 (61.8\%) succeeded in attaining CTP class A status after treatment. None of the patients with a baseline CTP score $>9$, i.e. CTP class $\mathrm{C}$, could attain a persistent compensated state, i.e. CTP A, despite DAA therapy in the follow-up period of 36 weeks.

Among patients with CTP class B cirrhosis or a low (< 15-18) MELD score, 35-61\% had improved MELD scores, whereas $22-33 \%$ had worsened MELD scores. Among patients with CTP class C cirrhosis or a high (> 15-18) MELD score, 81-87\% had improved MELD scores, whereas $13-33 \%$ had worsened MELD scores $[5,19,20]$.

The presence of SVR12 was not significantly associated with response to DAA treatment $(p=0.2)$. Only 2 patients in our study group who failed to maintain persistent CTP class A after treatment did not achieve SVR12. One patient was hospitalized twice during follow-up with tense ascites requiring large-volume paracentesis. The second patient also had a history of ascites twice in the follow-up period, requiring uptitration of diuretics.

On univariate analysis, factors that were significantly associated with improvement in liver function 
were serum albumin, serum bilirubin, and eGFR. However, on multivariate analysis, only a lower serum bilirubin was significantly associated with the attainment of Child A status after treatment.

The excellent efficacy and safety profile of DAAs has made antiviral therapy possible for patients with advanced liver disease. Treatment in such patients should be individualized, particularly if LT is an available option. In such cases, the challenge is to decide on therapy before or after LT.

In patients with decompensated cirrhosis, who are candidates for LT but access to it is difficult, DAA can be considered as the first line of therapy. Moreover, in patients who are LT eligible and have access to LT, but their MELD score is $<15$, treatment with DAA should be considered before LT [21]. Decompensated cirrhotics with a MELD score $>20$ and/or eGFR $<30 \mathrm{ml} / \mathrm{min} / 1.73 \mathrm{~m}^{2}$ should undergo liver transplant followed by DAA therapy as they have a lesser degree of improvement in hepatic function after HCV therapy, lower SVR rates (as low as 25\%) with limited safety profiles for DAAs [22, 23].

Existing data suggest that successful HCV therapy is associated with improvement of hepatic function in $20-60 \%$, which may lead to the delisting of patients (MELD $<16,16-20,>20$ have a delisting probability of $35 \%, 12 \%, 5 \%$ respectively) $[24,25]$. For the past twenty years, HCV has dominated transplant activity in North America and Europe, but with the availability of DAAs the trend is changing. As highlighted in the study by Belli and colleagues from the European Liver Transplant Registry (ELTR), listings for HCV declined from $22 \%$ in 2007 to $17 \%$ in 2017 [26]. These trends are similar to those of the United Network for Organ Sharing (UNOS), the equivalent registry in the US, where the percentage of patients with a diagnosis of $\mathrm{HCV}$ who were on the LT waiting list decreased from $37 \%$ in 2012 to $24 \%$ in 2016 [27]. So, it can be rightly said that the reign of the HCV as the "king" in liver transplantation is now over, with alcoholic liver disease (ALD) and non-alcoholic fatty liver disease (NAFLD) ready to assume the title! Much of the credit goes to DAA therapy for this achievement.

Dultz et al. in their retrospective analysis of 68 patients of HCV-associated advanced liver cirrhosis on antiviral therapy with pegylated interferon and ribavirin, reported decompensating events during follow-up in $36.8 \%$ of cases [28]. On multivariate analysis, MELD score was independently associated with hepatic decompensation ( $\mathrm{OR}=1.56,1.18-2.07, p=0.002)$. When the patients were grouped according to their baseline MELD scores, hepatic decompensation occurred in $22 \%, 59 \%$, and $83 \%$ of patients with a MELD score of
$6-9,10-13$, and $\geq 14$, respectively. Baseline factors that were significantly associated with attainment of a compensated state, i.e. Child-Pugh class A, were serum ALT and MELD score before starting DAA therapy $(p<0.05)$.

In our study, the number of patients with a MELD score $>15$ decreased from $21 \%$ to $14.5 \%$ after treatment with DAA. Two patients who failed to achieve SVR12 after DAA therapy had a MELD score $>15$, and they also had decompensating events in the post-treatment follow-up period.

We found that a majority of patients had a BE3A score of 2 (51.6\%), followed by 3 (25.8\%), 1 (19.4\%) and 4 in 3.2\%. All patients with a BE3A score of 4 had an improvement in liver function and attained a compensated state after treatment, i.e. CTP class A. However, 15/16 (93.7\%) patients with a BE3A score 3, 14/32 (43.7\%) with a score of 2 and only $3 / 12$ (25\%) with a score of 1 had Child-Pugh class A after treatment with DAA. El-Sherif et al. found that a BE3A score of $4-5$ was associated with a $75 \%$ chance of achieving CTP A, while those with a score of 0 or 1 were associated with a $<5 \%$ and $25 \%$ chance of achieving CTP A at 36 weeks, respectively [16]. BE3A scores of 3 and 4 were associated with $87.0 \%$ and $98.2 \%$ specificity of achieving a sustained CTP A status, whereas BE3A scores of 1 and 0 were associated with $92.3 \%$ and $99.6 \%$ specificity of not achieving a CTP A status.

A comparison of our composite model with BE3A score using ROC analysis revealed better results with our model, with an AUC value of 0.841 and 0.771 respectively. Our devised model takes into consideration demographic, clinical and biochemical parameters of all the study population, unlike the BE3A score, which used only clinical and biochemical variables. The sensitivity, NPV, and accuracy of our model were better than the BE3A score, whereas the latter had a better specificity and PPV in predicting response to therapy in this population. Moreover, using BMI as a parameter for predicting response, as used in the BE3A score, has serious implications, especially in patients with decompensated cirrhosis with huge ascites with pedal edema. In addition, BMI did not significantly alter the response to DAA in our population.

Dunn et al. in their recent prospective analysis of a similar group of patients found that the BE3A score failed to show significant benefit in predicting response to DAA therapy [29]. The authors found that PNPLA3 CG/GG genotypes could identify a subgroup of patients with decompensated HCV cirrhosis who had suboptimal clinical recovery despite achieving SVR. Genetic polymorphism might be associated with hepatic steatosis (rs738409 of PNPLA3) that may affect 
biochemical (MELD score) and clinical (CTP score) recovery following DAA treatment for $\mathrm{HCV}$ cirrhosis.

There are a few limitations to our study. Being a single-center study, the sample size is small. Moreover, a study with a much longer follow-up period might show a different perspective of the disease. A previous long-term study with sofosbuvir and ribavirin in patients with HCV cirrhosis and portal hypertension showed significant reductions in portal pressure only at 96 weeks after treatment [30]. Lens et al. demonstrated continual improvements in portal hypertension for up to 5 years after therapy, suggesting that a longer time interval is required for hepatic remodeling [31].

Direct or indirect assessment of change in liver fibrosis status in the post-treatment period was not quantified. As all our patients had ascites before starting therapy, baseline transient elastography was not performed. However, other indirect methods of fibrosis determination such as Acoustic Radiation Force Impulse (ARFI) can be considered in future studies for comparison of fibrosis in the pre- and post-treatment period. Lastly, our center lacked linkage to a transplant registry.

\section{Conclusions}

The present study shows that in patients with decompensated HCV cirrhosis of CTP class B and C, sofosbuvir-based combination DAA therapy with daclatasvir, ledipasvir or velpatasvir with or without ribavirin, is very effective and often leads to a remarkable clinical improvement with an excellent SVR rate of $98.6 \%$. It also attained compensated status from the baseline decompensated state in more than half of our patients. Our new model performed better than the BE3A score in predicting response to DAA therapy in this population, suggesting that this score can be improved. Moreover, our study showed that patients with a CTP score $>9$ and a MELD score $>15$ did not respond well to DAA therapy, and we suggest early LT followed by DAA therapy in such patients. However, long-term studies are encouraged before drawing a final conclusion. It should be remembered that such patients are always at risk of developing HCC and screening should be continued as per guidelines. Long-term benefits of DAA therapy need to be established.

\section{Disclosure}

The authors declare no conflict of interest.

\section{References}

1. Westbrook RH, Dusheiko G. Natural history of hepatitis C. J Hepatol 2014; 61: S58-68.

2. Foster GR, Irving W, Cheung MC, et al. Impact of direct antiviral therapy in patients with chronic hepatitis $\mathrm{C}$ and decompensated cirrhosis. J Hepatol 2016; 64: 1224-1231.

3. Poordad F, Schiff ER, Vierling JM, et al. Daclatasvir, sofosbuvir, and ribavirin combination for HCV patients with advanced cirrhosis or post-transplant recurrence: ALLY-1 study. J Hepatol 2015; 62: S261-262.

4. Charlton M, Everson GT, Flamm SL, et al. Ledipasvir and sofosbuvir plus ribavirin for treatment of HCV infection in patients with advanced liver disease. Gastroenterology 2015; 149: 649659.

5. Curry MP, O'Leary JG, Bzowej N, et al. Sofosbuvir and velpatasvir for $\mathrm{HCV}$ in patients with decompensated cirrhosis. $\mathrm{N}$ Engl J Med 2015; 373: 2618-2628.

6. Singh S, Facciorusso A, Loomba R, et al. Magnitude and kinetics of decrease in liver stiffness after antiviral therapy in patients with chronic hepatitis $\mathrm{C}$ : a systematic review and meta-analysis. Clin Gastroenterol Hepatol 2018; 16: 27-38.

7. Flemming JA, Kim WR, Brosgart CL, et al. Reduction in liver transplant wait-listing in the era of direct-acting antiviral therapy. Hepatology 2017; 65: 804-812.

8. Ahmed A, Gonzalez SA, Cholankeril G, et al. Treatment of patients waitlisted for liver transplant with all-oral direct-acting antivirals is a cost-effective treatment strategy in the United States. Hepatology 2017; 66: 56.

9. Jang JW, Choi JY, Kim YS, et al. Long-term effect of antiviral therapy on disease course after decompensation in patients with hepatitis B virus-related cirrhosis. Hepatology 2015; 61: 1809-1820.

10. Berenguer M, Ferrell L, Watson J, et al. HCV related fibrosis progression following liver transplantation: increase in recent years. J Hepatol 2000; 32: 673-684.

11. Gallegos-Orozco JF, Yosephy A, Noble B, et al. Natural history of post-liver transplantation hepatitis $\mathrm{C}$ : a review of factors that may influence its course. Liver Transpl 2009; 15: 1872-1881.

12. Samuel D, Forns X, Berenguer M, et al. Report of the monothematic EASL conference on liver transplantation for viral hepatitis (Paris, France, January 12-14, 2006). J Hepatol 2006; 45: 127-143.

13. Hatzakis A, Chulanov V, Gadano AC, et al. The present and future disease burden of hepatitis $\mathrm{C}$ virus (HCV) infections with today's treatment paradigm - volume 2. J Viral Hepat 2015; 22 (Suppl 1): 26-45.

14. Ahmed OA, Elsebaey MA, Fouad MH, et al. Outcomes and predictors of treatment response with sofosbuvir plus daclatasvir with or without ribavirin in Egyptian patients with genotype 4 hepatitis C virus infection. Infect Drug Resist 2018; 11: 441-445.

15. Buti M, Riveiro-Barciela M, Esteban R. Management of direct antiviral agent failures. J Hepatol 2015; 63: 1511-1522.

16. El-Sherif O, Jiang ZG, Tapper EB, et al. Baseline factors associated with improvements in decompensated cirrhosis after direct-acting antiviral therapy for hepatitis $\mathrm{C}$ virus infection. Gastroenterology 2018; 154: 2111-2121.e8.

17. American Association for the Study of Liver Diseases and the Infectious Diseases Society of America. HCV guidance: recommendations for testing, managing, and treating hepatitis $\mathrm{C}$. http:// www.hcvguidelines.org/. 
18. Puri P, Saraswat VA, Dhiman RK, et al. Indian National Association for Study of the Liver (INASL) Guidance for Antiviral Therapy Against HCV Infection: Update 2016. J Clin Exp Hepatol 2016; 6: 119-145.

19. Fernandez Carrillo C, Lens S, Llop E, et al. Treatment of hepatitis $C$ virus infection in patients with cirrhosis and predictive value of model for end-stage liver disease: analysis of data from the Hepa-C registry. Hepatology 2017; 65: 1810-1822.

20. Manns M, Samuel D, Gane E, et al. Ledipasvir and sofosbuvir plus ribavirin is patients with genotype 1 or 4 hepatitis $C$ virus infection and advanced liver disease: a multicentre, open label, randomised, phase 2 trial. Lancet Infect Dis 2016; 16: 685-697.

21. Belli LS, Duvoux C, Berenguer M, et al. ELITA consensus statements on the use of DAAs in liver transplant candidates and recipients. J Hepatol 2017; 67: 585-602.

22. Modi AA, Nazario H, Trotter JF, et al. Safety and efficacy of simeprevir plus sofosbuvir with or without ribavirin in patients with decompensated genotype 1 hepatitis $\mathrm{C}$ cirrhosis. Liver Transpl 2016; 22: 281-286.

23. Bunchorntavakul C, Reddy KR. HCV therapy in decompensated cirrhosis before or after liver transplantation: a paradoxical quandary. Am J Gastroenterol 2018; 113: 449-452.

24. Bunchorntavakul C, Reddy KR. Treat chronic hepatitis C virus infection in decompensated cirrhosis - pre- or post-liver transplantation? The ironic conundrum in the era of effective and well-tolerated therapy. J Viral Hepat 2016; 23: 408-418.

25. Belli LS, Berenguer M, Cortesi PA, et al. Delisting of liver transplant candidates with chronic hepatitis $\mathrm{C}$ after viral eradication: a European study. J Hepatol 2016; 65: 524-531.

26. Belli L, Perricone G, Adam R, et al. Impact of DAAs on liver transplantation: major effects on the evolution of indications and results. An ELITA study based on the ELTR registry. J Hepatol 2018; 69: 810-817.

27. Cholankeril G, Ahmed A. Alcoholic liver disease replaces hepatitis $\mathrm{C}$ virus infection as the leading indication for liver transplantation in the United States. Clin Gastroenterol Hepatol 2017; 16: 1356-1358.

28. Dultz G, Seelhof M, Herrmann E, et al. Baseline MELD score predicts hepatic decompensation during antiviral therapy in patients with chronic hepatitis $\mathrm{C}$ and advanced cirrhosis. PLoS One 2013; 8: e71262.

29. Dunn W, Vittal A, Zhao J, He J, et al. PNPLA3 gene predicts clinical recovery after sustained virological response in decompensated hepatitis C cirrhosis. BMJ Open Gastroenterol 2019; 6: e000241.

30. Afdhal N, Everson GT, Calleja JL, et al. Effect of viral suppression on hepatic venous pressure gradient in hepatitis $\mathrm{C}$ with cirrhosis and portal hypertension. J Viral Hepat 2017; 24: 823-831.

31. Lens S, Rincon D, Garcia-Retortillo M, et al. Association between severe portal hypertension and risk of liver decompensation in patients with hepatitis $\mathrm{C}$, regardless of response to antiviral therapy. Clin Gastroenterol Hepatol 2015; 13: 1846-1853. 